\title{
Analysis of the optimal time delay for Reconstruction of Phase Space of VCG signals to identify anterior wall infarction
}

\author{
Rafael Duarte de Sousa, José Raimundo Barbosa, Ana Beatriz S. N. R. de Oliveira and Carlos Danilo M. Regis.
}

\begin{abstract}
Acute Myocardial Infarction (MI) can be detected through Reconstructed Phase Space (RPS) parameters. The RPS is dependent on a time delay $(\tau)$ that can be a fixed or an optimal value based on the mutual information between a vectorcardiogram lead and its delayed version. This paper aims to assess the effect of an optimized time-delay in the detection of MI in the anterior wall using RPS parameters. For optimal $\tau$ values the sensitivity, specificity, accuracy and AUC obtained were: $64.7 \%, 63.2 \%, 57.8 \%$ and $62.3 \%$, respectively; while for a fixed $\tau$ value were, respectively, $81.3 \%, 74.5 \%, 64.9 \%$ and $70.1 \%$.
\end{abstract}

Keywords-Reconstructed Phase Space, Anterior Myocardial Infarction, Deep Neural Network, Vectorcardiogram.

\section{INTRODUCTION}

According to the World Health Organization, cardiovascular diseases, including Myocardial Infarction (MI), also known as heart attack, are the world's leading cause of death [1]. The diagnosis of MI is usually done using the 12-lead electrocardiogram (ECG) exam or the vectorcardiography (VCG) [2]. Both of these signals can have their Phase Space reconstructed by time-delay methods. The delay $(\tau)$ applied on a signal can be either a fixed value used for all signals in a set as used in [3] or an optimized value for each signal.

The Reconstructed Phase Space (RPS) representation is used to determine how chaotic a certain signal is and also highlights features hidden in the original signal. This method was first applied to ECG signals due to the more chaotic behavior in pathological signals so it could be used to classify them.

Since the RPS is highly dependent on the $\tau$ value assigned to the signal and, to our knowledge, there are no studies concerning the use of an optimal $\tau$ and its effect in the identification of myocardial infarction, this paper aims to compare the performance of deep neural networks, one trained using the parameters obtained from a fixed $\tau$ value of $0.01 \mathrm{~s}$ for the training dataset and another trained using optimized $\tau$ values for each signal of the same dataset to verify if there is a performance increase for optimized values.

\section{VCG RECONSTRUCTION USING KORS METHOD}

The VCG signals can be reconstructed from the 12-lead ECG using a regression matrix. In this method, one can obtain

Rafael Duarte, e-mail: rafaelds57@gmail.com; José Raimundo Barbosa, email: jsraimundob@gmail.com; Ana Beatriz Nogueira, email: abeatriz.engeletrica@gmail.com; Carlos Danilo Regis, e-mail: danilo.regis@ifpb.edu.br. Federal Institute of Paraíba, João Pessoa - Paraíba. a vector containing the VCG orthogonal leads $V_{X}, V_{Y}$, and $V_{Z}$ by the product of a vector of the leads $I, I I, V 1, V 2, V 3$, $V 4, V 5, V 6$ and a matrix containing the linear combination coefficients. These coefficients are the ones for the Linear Regression method presented in [4] This relation is resumed in Equation 1:

$$
\left[\begin{array}{c}
V_{X} \\
V_{Y} \\
V_{Z}
\end{array}\right]=\left[\begin{array}{lll}
a_{11} & \ldots & a_{18} \\
a_{21} & \ldots & a_{28} \\
a_{31} & \ldots & a_{38}
\end{array}\right] \cdot\left[\begin{array}{c}
I \\
\vdots \\
V 6
\end{array}\right]
$$

\section{Reconstructed Phase Space}

A phase space is a space that contains all the possible states of a system. Using this tool is possible to evaluate how chaotic is a system and find information in systems that apparently behave chaotically.

The reconstruction of the Phase Space can be done using a temporal series of a variable such as an ECG or VCG lead. Generally, the n-dimensional phase space $\left(X_{n}\right)$ of a signal is given by the Equation 2, as defined by [5].

$$
X_{n}=\left[\begin{array}{llll}
x_{n} & x_{n-\tau} & \cdots & x_{n-(d-1) \tau}
\end{array}\right], \quad n=(1+(d-1) \tau) \ldots N .
$$

Where $x_{n}$ is the n-th point in the time series that goes up to $\mathrm{N}, \tau$ is the delay applied to the signal and $\mathrm{d}$ is the dimension of the phase space.

For an ECG/VCG signal, this process consists of plotting the original signal, usually denoted as $(x(t))$, in the $\mathrm{X}$-axis and its delayed version $(x(t+\tau))$ in the Y-axis, generating an image of the RPS, as performed by [6].

\section{A. Optimal time delay value}

In order to reduce the correlation between the signal and its delayed version, the optimal $\tau$ is defined as the first local minimum in the mutual information (MInf) curve [7]. The MInf is a concept from Information Theory that is used to measure the dependence of two variables. It is based on other concepts from Information Theory such as the entropy of a random variable $(\mathrm{H}(\mathrm{X}))$ and the conditional entropy of this random variable given another random variable $(\mathrm{H}(\mathrm{X} \mid \mathrm{Y}))[8]$.

The MInf, given by $I$, represents the amount of information for a variable when another variable is known. Considering the random variables $X$ and $Y$, the MInf is given by Equation 3 [8]. 


$$
I(X ; Y) \equiv H(X)-H(X \mid Y)=H(Y)-H(Y \mid X)
$$

In this work, the MInf is estimated using the algorithm described by [9]. With it, the MInf is obtained for 100 different $\tau$ values, where the interval of these values is the sampling interval. Considering that, for $(\mathrm{n}=1,2, \ldots, 100)$, the optimal $\tau$ was defined as the first $\tau$ value that satisfies the following relationship:

$$
I(x(t), x(t+\operatorname{tau}[n]))<I(x(t), x(t+\operatorname{tau}[n+1]))
$$

\section{Methods}

The 12-lead ECG signals and their respective classes were obtained from the PTB-XL, a database from The National Metrology Institute of Germany available in [10], which has ECG signals with the sampling frequency of $500 \mathrm{~Hz}$. In this work, only the classes "Normal" and "Anterior Wall Myocardial Infarction"(AMI) were used. For each of them, 1019 signals were chosen.

Each 12-lead ECG signal had the same processing techniques used in [11] applied to it, generating the RPS images for each VCG lead with $512 \times 512$ pixels using the $\tau_{1}=0.01 \mathrm{~s}$ and $\tau_{2}=0.02 \mathrm{~s}$. RPS images were also generated using the optimized $\tau_{1}$ values and with $\tau_{2}=2 * \tau_{1}$. For all the RPS generated the parameters described by [11] were calculated using a block size of 20x20.

These these RPS parameters obtained from the images were used to train two train deep neural networks (DNN) that received the all 15 parameters (five parameters for each VCG lead). The DNNs had 10 hidden layers with [32, 256, 256, 128 , $128,64,32,16,8,4]$ neurons using the activation function ReLU and with a dropout of 0.1 , while the output neuron used the sigmoid function. The training lasted 5000 iterations with a learning rate of 0.001 using the ADAM optimizer. To determine the training and cross-validation sets the stratified $\mathrm{K}$-fold using $k=5$ was employed. The sensitivity, specificity, accuracy, and AUC (area under the Receiver Operating Characteristic curve) were obtained.

\section{RESUlts}

The results obtained after the training of the DNN are shown in Table I. These values were compared in order to determine whether parameters obtained from RPS generated using the optimized $\tau$ could train a classification with higher performance.

TABLE I

RESULTS FOR THE CLASSIFICATION USING A FIXED AND AN OPTIMIZED $\tau$.

\begin{tabular}{|l|l|l|l|l|}
\hline & Sensitivity & Specificity & Accuracy & AUC \\
\hline Fixed $\tau$ & 0.8137 & 0.7451 & 0.6495 & 0.7019 \\
\hline Optimal $\tau$ & 0.6471 & 0.6324 & 0.5784 & 0.6230 \\
\hline
\end{tabular}

These results suggest that the fixed $\tau$ values obtained empirically by [3] and later used by [12] lead to better performance compared to the optimal $\tau$. There are, however, several limitations in this methodology. The first limitation is that while the optimal delay was determined with the MInf curve, the corresponding delay for the RPS to be compared was fixed as 2 times the optimal delay, which may have lead to worse performance.

The second one is that only the block size of 20x20 was used. While this block size led to better results for a fixed $\tau$, according to [12], this not be the case for an optimal $\tau$.

Lastly, only one type of infarction was used in the comparison of these two methods, which might not give an entire idea of the effect of the optimal $\tau$.

\section{CONCLUSION}

This work consists of an initial study of the effects of the optimized delay used in the reconstruction of the phase space of vectorcardiogram leads in the detection of myocardial infarction in the anterior wall, something not evaluated until this work.

While, since the fixed delay led to a better classification performance, these results suggest that the optimal $\tau$ reduces the classification performance, further research is needed to determine whether this applies to all classes of infarction and to evaluate the most adequate block size and second value of optimal delay.

\section{ACKNOWLEDGMENT}

The authors would like to thank the PPGEE/IFPB and the CNPq for all the financial support.

\section{REFERENCES}

[1] W. H. Organization, "Cardiovascular diseases (cvds)s," Available in: https://www.who.int/en/news-room/fact-sheets/detail/cardiovasculardiseases-(cvds), 2017, acessed: August 2021

[2] R. Walsh, Hurst's the Heart Manual of Cardiology. McGraw-Hill Education, 2012.

[3] M. Roopaei, R. Boostani, R. R. Sarvestani, M. A. Taghavi, and Z. Azimifar, "Chaotic based reconstructed phase space features for detecting ventricular fibrillation," Biomedical Signal Processing and Control, vol. 5, no. 4, pp. 318-327, 2010.

[4] J. Kors, G. Van Herpen, A. Sittig, and J. Van Bemmel, "Reconstruction of the frank vectorcardiogram from standard electrocardiographic leads: diagnostic comparison of different methods," European Heart Journal, vol. 11, no. 12, pp. 1083-1092, 1990.

[5] M. W. Zimmerman, R. J. Povinelli, M. T. Johnson, and K. M. Ropella, "A reconstructed phase space approach for distinguishing ischemic from non-ischemic st changes using holter ecg data," in Computers in Cardiology, 2003. IEEE, 2003, pp. 243-246.

[6] A. Amann, R. Tratnig, and K. Unterkofler, "Detecting ventricular fibrillation by time-delay methods," IEEE Transactions on Biomedical Engineering, vol. 54, no. 1, pp. 174-177, 2006.

[7] A. M. Fraser and H. L. Swinney, "Independent coordinates for strange attractors from mutual information," Physical review A, vol. 33, no. 2, p. 1134, 1986.

[8] D. J. MacKay and D. J. Mac Kay, Information theory, inference and learning algorithms. Cambridge university press, 2003.

[9] A. Kraskov, H. Stögbauer, and P. Grassberger, "Estimating mutual information," Physical review E, vol. 69, no. 6, p. 066138, 2004.

[10] P. Wagner, N. Strodthoff, R.-D. Bousseljot, D. Kreiseler, F. I. Lunze, W. Samek, and T. Schaeffter, "Ptb-xl, a large publicly available electrocardiography dataset," Scientific Data, vol. 7, no. 1, pp. 1-15, 2020.

[11] C. M. Costa, I. S. Silva, R. D. de Sousa, R. A. Hortegal, and C. D. M. Regis, "The association between reconstructed phase space and artificial neural networks for vectorcardiographic recognition of myocardial infarction," Journal of electrocardiology, vol. 51, no. 3, pp. 443-449, 2018.

[12] C. Costa, "Identificação do infarto do miocárdio por meio da análise do cardiograma vetorial e da reconstrução do espaço de fases," Master's thesis, Instituto Federal da Paraíba, 2017. 